\title{
Distritos creativos en la Ciudad de México en la segunda década del siglo XXI
}

Mexico City's Creative Districts at the Second Decade of the XXI Century

Distritos criativos na Cidade de México na segunda década do século XXI

\section{Alejandro Mercado Celis*}

Recibido: 26 de febrero de 2015

Aprobado: 29 de septiembre de 2015

Doi: dx.doi.org/10.12804/territ34.2016.08

Para citar este artículo

Mercado Celis, A. (2016). Distritos creativos en la Ciudad de México en la segunda década del siglo XxI. Territorios, 34, 183-213. Doi: dx.doi.org/10.12804/territ34.2016.08
* Doctor en Planeación Urbana por la Universidad de California, Los Ángeles. Profesor-investigador Titular " $C$ " de tiempo completo, Universidad Autónoma Metropolitana, Unidad Cuajimalpa. Departamento de Ciencias Sociales. Correo electrónico: alejandromer@gmail.com 
Palabras clave

Desarrollo urbano, distritos creativos, clusters económicos, distritos urbanos, industrias creativas, Ciudad de México.

Keywords

Urban development, creative districts, economic clusters, urban districts, creative industries, Mexico

City.

Palavras-chave

Desenvolvimento urbano, Distritos Criativos, Clusters económicos, distritos urbanos, indústrias criativas, Cidade do México.

\section{territarias 34}

\section{RESUMEN}

La aglomeración de empresas culturales/creativas y establecimientos de consumo especializado en distritos urbanos marcados por un proceso de rápida transformación urbana se ha identificado como 'distritos creativos' y se asume que son clusters económicos, en el sentido en que forman un sistema aglomerado de empresas especializadas y su correspondiente mercado laboral. Para esta investigación, estos distritos son una parte visible e idealizada de un cluster económico más grande que opera en una escala metropolitana y que incluye zonas, empresas y trabajos diversos. Se inicia este texto discutiendo la problemática conceptual y metodológica de llevar el concepto de cluster económico a la escala geográfica intraurbana. Con ello, se confronta la idea de 'distritos creativos' como espacios económicos funcionales y separables del resto de la economía metropolitana donde se ubican. Posteriormente, se aplican los métodos que comúnmente se utilizan en los estudios urbanos sobre industrias creativas para identificar posibles distritos creativos en la Ciudad de México. Los distritos identificados son contrastados en sus estructuras y especializaciones económicas para determinar si hay indicios de que, efectivamente, sean espacios económicos funcionales diferenciables del resto de la zona metropolitana. Se concluye argumentando que los datos encontrados sustentan la existencia de un solo cluster económico metropolitano y no de pequeños clusters.

\section{ABSTRACT}

The agglomeration of cultural / creative industries and specialized retail stores in urban districts marked by a rapid urban transformation process has been identified as "Creative Districts". It has been assumed that these districts are economic clusters in the sense of being an economic system formed by specialized companies and their corresponding labor market. From our point of view these districts are only a visible part of a larger economic cluster operating at the metropolitan scale which includes many different areas, enterprises and jobs. In this paper we discuss the conceptual and methodological problems of bringing the concept of economic cluster or industrial district to the intra-urban geographic scale. In order to confront the idea of 'creative districts' as functional economic areas separable from the rest of the metropolitan economy, we apply the methods commonly used in urban studies on creative industries to identify possible creative districts within Mexico City. The identified districts are contrasted in their economic structures to determine if there are indications that indeed they are differentiable and functional economic clusters from the rest of the metropolitan area. We conclude by arguing that the data found sustain the existence of a single metropolitan economic cluster and no small clusters in urban districts.

\section{RESUMO}

A aglomeração de empresas culturais/criativas e estabelecimentos de consumo especializado em distritos urbanos marcados por um processo de rápida transformação urbana se tem identificado como "Distritos Criativos" e se assume que são clusters económicos no sentido de formar um sistema aglomerado de empresas especializadas e seu correspondente mercado laboral. Desde nosso ponto de vista estes distritos são uma parte visível e idealizada de um cluster económico mais grande que opera em uma escala metropolitana e que inclui zonas, empresas e trabalhos diversos. Iniciamos este texto discutindo a problemática conceitual e metodológica de levar o conceito de cluster 
económico à escala geográfica intra-urbana. Com isso confrontamos a ideia de "distritos criativos" como espaços económicos funcionais e separáveis do resto da economia metropolitana onde se localizam. Posteriormente, aplicamos os métodos que comumente se utilizam nos estudos urbanos sobre indústrias criativas para identificar possíveis distritos criativos na Cidade do México. Os distritos identificados são contrastados em suas estruturas e especialização económicas para determinar se há indícios de que efetivamente sejam espaços económicos funcionais diferenciáveis do resto da zona metropolitana. Concluímos argumentando que os dados encontrados sustentam a existência de um só cluster económico metropolitano e não de pequenos clusters.

\section{Introducción. Economía creativa y las grandes metrópolis}

Los sectores de servicios y comercio conectados con la economía creativa han tomado un papel relevante en los procesos económicos y de transformación urbana en las grandes metrópolis del mundo. La Ciudad de México y su zona metropolitana $(\text { ZMVM })^{1}$ es un buen ejemplo de esto, sectores vinculados a la producción de bienes y servicios con un alto contenido estético y simbólico han crecido con fuerza; en particular, el cluster de la publicidad presenta una alto nivel de concentración en la ciudad, pero también áreas como la arquitectura, el diseño en todas sus áreas y áreas tradicionales como la producción editorial o nuevos como el diseño web son parte del panorama económico actual del ZMVM. En cuanto a la ubicación de estas empresas dentro de la ciudad, se puede ver que se insertan y entremezclan en el entramado urbano, conviviendo con usos residenciales, de consumo, de entretenimiento, etc. "Las industrias de productos culturales, como nunca antes funden el aparato de producción y el medio ambiente social y urbano" (Power \& Scott, 2004, p. 8).
A la aglomeración de empresas y trabajadores creativos en algunas zonas de las ciudades en las que se coincide con contextos urbanos estéticamente atractivos, así como con una densidad importante de negocios gastronómicos y de consumo especializado se le identifica como 'distritos creativos'. En el caso de la Ciudad de México y su zona metropolitana, recientemente la zona de las colonias Roma-Condesa ha capturado la imagen de un distrito creativo (Mercado Celis, 2012; Moreno, Mercado \& Félix, 2011; Pita, 2015). Esta percepción se basa, por una parte, en la aglomeración de profesionales, trabajadores creativos y de artistas en general, $y$, por otra, en la concentración de una oferta gastronómica y de consumo diversificada y especializada (Delgadillo, 2014; Gómez, 2015), en un contexto urbano de construcciones y espacios públicos estéticamente atractivos y en una ubicación central privilegiada. La identificación de esta zona como distrito creativo se debe también a que los 'comercios creativos' (galerías, tiendas de diseño, ropa, restaurantes de nueva gastronomía, etc.) han implementado campañas de publicidad que promueven la idea de un Corredor
${ }^{1}$ La Zona Metropolitana del Valle de México está formada por 16 delegaciones del Distrito Federal, más 59 municipios del Estado de México y 1 municipio del Estado de Hidalgo. 
${ }^{2}$ Los elementos que diferencian a los distritos industriales de los clusters económicos es que los primeros operan bajo un sistema basado en pequeñas empresas con un sistema de gobernanza no jerárquico, mientras que el concepto de cluster se refiere a cualquier sistema de gobernanza de un sistema económico aglomerado.

${ }^{3}$ Los sectores creativos incluyen disciplinas culturalestradicionales - música, pintura, danza, teatro, etc.-, y actividades cuyo producto tiene un alto contenido estético/simbólico —publicidad, moda, videojuegos, edición de páginas web, entre otros-. En este trabajo se hace referencia a la definición amplia se sectores creativos y se usa el término cultural como sinónimo.

territarias 34
Cultural Roma-Condesa (Corredor Roma Condesa, 2015).

Ante la aparición de este y otros distritos, surge la pregunta de si estas aglomeraciones de empresas creativas, consumo especializado en contextos urbanos marcados por un proceso de rápida transformación (reutilización de edificios, expansión comercial, construcción intensa y revalorización del precio suelo, etc.) constituyen un cluster económico (sistema aglomerado de empresas especializadas y su correspondiente mercado laboral) $\mathrm{o}$, por el contrario, zonas como la Roma-Condesa son en realidad una parte visible e idealizada de un cluster económico más grande que opera a una escala metropolitana y que incluye zonas, empresas y trabajos de mayor y menor glamour. Para responder esta pregunta, se discutirá primero la problemática conceptual y metodológica de llevar el concepto de cluster económico o distrito industrial a la escala geográfica intraurbana. Con ello, se confronta el discurso de estrategias de desarrollo urbano basadas en 'distritos creativos' como espacios económicos funcionales y separables del resto de la economía metropolitana donde se ubican. En segundo lugar, se aplican los métodos que comúnmente se utilizan en los estudios urbanos sobre industrias creativas para analizar la distribución espacial de estos sectores en el ZMVM e identificar posibles distritos creativos. Los distritos identificados son contrastados en sus estructuras y especializaciones económicas para determinar si hay indicios de que efectivamente sean espacios económicos funcionales diferenciables del resto de la zona metropolitana. Finalmente, se concluye argumentando que los datos muestran la existencia de una gran aglomeración económica a nivel metropolitano con algunos rasgos distintivos entre las zonas urbanas donde se ubican, principalmente entre la zona central y la periferia.

\section{Distritos creativos: cluster económico creativo o distrito urbano creativo}

En esta sección se contrastan dos corrientes de estudios sobre las industrias creativas y su aglomeración espacial, uno proveniente de los estudios urbanos y el otro de la geografía económica. En primer lugar, se resumen las características de cada corriente en cuanto a su definición de 'distrito/cluster creativo', la escala territorial predominante y la metodología empleada en su identificación. En segundo lugar, se comparan las diferencias y similitudes entre ambas corrientes.

En el área de estudios urbanos, el uso del término distrito o cluster ${ }^{2}$ creativo y otros sinónimos de aglomeración como zona, centro o corredor, se refieren a la aglomeración intraurbana de organizaciones, empleo y/o infraestructura pública o privada vinculadas a sectores creativos $^{3}, \mathrm{O}$ bien a una alta concentración de artistas. Por ejemplo, Frost-Kumpf (1998) señala que "un distrito cultural es un área de una ciudad con reconocimiento, nombrada y con usos mixtos en donde una alta concentración de instalaciones culturales sirven 
como ancla de atracción" (p. 7). Otros autores enfatizan la aglomeración de empresas creativas por sobre la de instalaciones o infraestructura cultural (Smit, 2011), o si la aglomeración de empresas está dominada por organizaciones burocráticas, privadas o comunitarias (Zarlenga, Ulldemolins \& Morato, 2013). Otras definiciones denotan si el origen de la aglomeración en los distritos es el producto de políticas públicas o de un proceso espontáneo, en cuyo caso se les denomina 'distritos culturales naturales' (Stern \& Seifert, 2007). Finalmente, Zukin y Braslow (2011), implícitamente denominan 'distritos creativos' a las zonas en donde habitan mayoritariamente artistas. El énfasis de todas estas definiciones está en la aglomeración de actividades culturales/ creativas y no entran cuestiones de estructura o funcionamiento económico.

Otro punto en común en la literatura de la corriente de estudios urbanos es usar la escala geográfica del barrio o su equivalente 'distrito'. Frost-Kumpf(1998) indica que la escala geográfica de los distritos es "usualmente menor de cien cuadras" (p. 10). Los términos vecindario o barrio lo usan la mayoría de los autores, denotando áreas no muy extensas dentro de las ciudades y con un carácter sociocultural distintivo. $\mathrm{Al}$ gunos ejemplos que usa la literatura son $\mathrm{El}$ Rabal en Barcelona, y Greenwitch Village y sOHO, en Nueva York.

En cuanto a los métodos de identificación en los textos de la corriente de estudios urbanos, hay que resaltar que la mayoría no utiliza ninguna metodología para su identificación y/o para delimitar su extensión física. Solamente se encuentran dos textos que identificaron los distritos bajo estudio por medio de una metodología explícita. En el primer caso, se utiliza un índice de densidad compuesto por el número de empresas, organizaciones sin fines de lucro y artistas a lo largo de la ciudad y se complementa con un índice de asistencia a eventos culturales (Stern \& Seifert, 2007). En el segundo texto se identifican distritos solo con densidad de empresas creativas, y las fronteras de cada distrito se trazan utilizando una perspectiva subjetiva de la extensión del distrito (Smit, 2011). Como ya se mencionó, la mayoría de los trabajos revisados toman distritos que son popularmente reconocidos como áreas de concentración de actividades culturales o de artistas. Un buen ejemplo de esto son los trabajos de Zukin $(2009,2011)$ quien ha trabajado los barrios de artistas en Nueva York y su efecto gentrificador. Otros tantos estudios parten de áreas designadas por una autoridad gubernamental como 'distritos creativos (u otro sinónimo)' y que se convierten en zonas de intervención, a veces con una autoridad coordinadora, para el impulso de la regeneración urbana vía actividades culturales (Irani, 2014).

En contraposición de los estudios urbanos sobre distritos creativos, la corriente de literatura de geografía económica sobre clusters y distritos creativos utiliza los modelos teóricos desarrollados alrededor del trabajo de Porter (1998) sobre clusters económicos, del trabajo de los italianos sobre distritos industriales y de los geógrafos económicos-urbanos sobre regiones. Se debe territarias 34

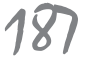


recordar que la definición de cluster/distrito industrial incluye los siguientes elementos: un conjunto de empresas aglomeradas territorialmente en un campo específico de producción, las actividades económicas complementarias y de soporte vinculadas a ellas, las organizaciones de apoyo tales como educativas y regulatorias, etc., y las instituciones formales e informales que permiten y orientan la coordinación del sistema aglomerado (Becattini, 1989, 2002; Porter, 1998; Storper \& Scott, 2003). La concentración de estos sistemas se explica por la existencia de economías de aglomeración que se expresan en diversas externalidades que benefician a las empresas y a los trabajadores y que, en un proceso evolutivo de retroalimentación, van generando y reforzando la aglomeración. Las economías de aglomeración se expresan en tres aspectos, uno es la existencia de una infraestructura común y la oferta localizada de algunos servicios y componentes especializados; otro es la generación de un pool de fuerza de trabajo especializada y el último, la transferencia/circulación de conocimiento en la aglomeración que facilita el aprendizaje y la innovación tecnológica (Strange, 2008).

Bajo este enfoque, un cluster/distrito creativo es un cluster/distrito industrial orientado a la producción de bienes o servicios creativos (Lazzeretti, 2013; Russo, 2000; Sacco \& Blessi, 2008; Scott \& Lerriche, s. f.; Van den Berg, Braun \& Van Winden, 2001). En el caso de los estudios europeos, se hace particular énfasis en la presencia de recursos históricos y naturales
(Lazzeretti, 2008). Hay que agregar a esta definición general particularidades derivadas del caso específico de la producción de bienes o servicios culturales tales como diversidad de tamaños de las empresas participantes con un predominio de pequeñas empresas; fragmentación y especialización en el proceso productivo, lo que genera interdependencias importantes; extrema flexibilización en las empresas y en el mercado laboral, lo que se expresa en una tendencia a la organización por proyectos y al uso de free lance; una condición de continua "novedad" en el diseño o contenido del producto/servicio, e interdependencia o retroalimentación con el contexto sociocultural y estético de la ciudad donde se aglomera el distrito (Power \& Scott, 2004).

A diferencia de la corriente de estudios urbanos, en la geografía económica, la escala geográfica de un cluster es la de una ciudad completa y su región de influencia inmediata, también conocida como zona metropolitana o ciudad-región (Scott, 2001). Aunque hay controversia en cuanto a la escala supraurbana, es decir la posibilidad de que se den clusters económicos que cubran más de una zona metropolitana, o uno o varios estados (Martin \& Sunley, 2003) y al uso mismo del término "región" (López \& Rebeca, 2012), la mayoría de los casos ejemplares que ha usado la literatura denotan el predominio de la dimensión regional (una ciudad central y su área de influencia inmediata o zona metropolitana), como es el caso del Valle del Silicón (alta tecnología) y de Los Ángeles (entretenimiento). Teóricamente, la definición más 
influyente en la literatura poscluster es la presentada por Storper y Venables en la que se afirma que la base esencial de las economías de aglomeración es la posibilidad del encuentro cara a cara, de forma cotidiana y repetida (Storper \& Venables, 2004). Esta condición sugiere una escala no mayor a la de zona metropolitana, espacio en el cual es posible tener este tipo de encuentro de forma cotidiana.

En la literatura de clusters/distritos económicos, específicamente de sectores creativos, la escala geográfica que domina también es la de ciudades enteras, ciudades y su región y regiones formadas por varias ciudades; la escala intraurbana no aparece. Algunos ejemplos: Arnaboldi et al., estudia un cluster cultural-turístico identificado como un área natural montañosa en el norte de Italia llamada Valsassina que incluye 28 municipalidades dentro de la provincia de Lecco (Arnaboldi \& Spiller, 2011). Otro estudio de cluster, en este caso, enfocado a la industria musical es el de Piedemonte, en Italia. La Región de Piedemonte incluye a la ciudad de Turín y, en conjunto, se habla de 4.5 millones de habitantes (Mizzau \& Montanari, 2008). De los trabajos de Allen Scott (2005) en el área de economía cultural se destaca como ejemplo su libro sobre Hollywood, en el que la escala de análisis comprende al conjunto de la zona metropolitana de Los Ángeles en un periodo histórico que inicia con el siglo Xx. En Latino América se citan los trabajos de Mochi Alemán sobre la industria del software que emplean la escala metropolitana usando los casos de Mérida, México y Rosario, Argen- tina (Mochi, 2010), el de Mercado Celis sobre el distrito Industrial de Tlaquepaque y Tonalá, en la Zona Metropolitana de Guadalajara, México (Mercado, 2006) y el trabajo de Turini, que, además de emplear una escala territorial regional, utilizando el caso de la ciudad de Santiago de Chile, analiza la escena de la música experimental y demuestra que está no ocurre en un solo espacio de la ciudad sino que sucede en una diversidad de espacios que en el transcurso del tiempo van cambiando y recomponiéndose (Tironi, 2012).

En cuanto a la metodología de identificación, en la corriente de geografía económica, la mayoría de los autores parten de clusters/distritos ya identificados por autoridades locales o parten de casos en los que el reconocimiento es evidente por su continuidad histórica o por su reconocimiento público o por contener empresas importantes. Un buen ejemplo de estudios que parten de clusters, identificados por las autoridades locales, es el trabajo de Van den Berg (2001), quien recibe de autoridades de las ciudades miembro de la -Red de Eurociudades- una lista de clusters que ellos "percibían como las fuentes más prometedoras de crecimiento económico" (p. 186). En la misma lógica, Arnaboldi trabaja el distrito cultural de Valessina en Italia a partir de la zona definida por las autoridades de la Comunidad de las Montañas de Vlaessina, la Provincia de Lecco y la Cámara de Comercio de Lecco (Arnaboldi \& Spiller, 2011). En el caso de clusters reconocidos, está el trabajo de Sacco y Blessi (2008), quienes usan en su texto ejemplos renom- territarias 34

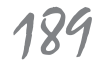


brados como Austin, Texas, sin emplear ninguna metodología que lo justifique como cluster. En esta misma línea, el artículo de Mizzau y Montanari (2008) justifica la selección del cluster de la música en Piedmont argumentando que "Piedmont se caracteriza por la presencia de algunas disqueras independientes importantes, alberga algunas de las bandas italianas más conocidas y recientemente ha ganado visibilidad nacional e internacional en el campo de la música electrónica a través de un proyecto impulsado por las autoridades regionales locales" (p. 652).

Hay que mencionar los estudios que intentan validar/evaluar clusters creativos aunque no identificarlos. Lazzareti (2008) ha desarrollado una metodología para evaluar el grado de estructuración de un distrito analizando su dotación de recursos, los actores involucrados, las relaciones entre los actores, y su comportamiento. Otro caso es el de la investigación de Russo (2000) que parte de destinos turísticos autoidentificados por autoridades gubernamentales, pero los somete a un análisis para confirmar si son o no clusters. Los criterios que emplea para evaluarlos son proximidad (difusión, transferencia, recursos comunes), flexibilidad (aprendizaje), actividades medianas y pequeñas (flexibilidad externa), cooperación (alianzas estratégicas) y competitividad (competencia suficiente para impulsar alianzas).

Hasta aquí se han presentado las dos corrientes principales que utilizan el concepto de cluster/distrito creativo: la geografía económica y los estudios urbanos.
Esta revisión permite definir sus principales diferencias y similitudes. La geografía económica enfatiza los aspectos funcionales y de especialización económica, los estudios urbanos enfatizan la desigual concentración de empresas, infraestructura y artistas dentro de las ciudades. En términos de escala geográfica, la primera tiende a concentrarse en unidades territoriales iguales o mayores a una ciudad, la segunda en la escala intraurbana. Ambas líneas de investigación tienden a partir de casos conocidos o aceptados como válidos por su reconocimiento público o por autoridades locales. Como se puede ver, los enfoques no son explicaciones en competencia, por el contrario, al referirse a procesos y escalas diferentes pueden ser complementarios. No obstante lo anterior, el reconocimiento de esta posible complementariedad y de la necesidad de tener bien definidas las diferencias conceptuales entre ambas aparecen en muy pocos trabajos académicos (una excepción es la monografía de la OCDE (2005) sobre distritos creativos. Por el contrario, existen algunos autores que mezclan conceptualmente ambos enfoques en la escala intraurbana. A continuación se revisa esta literatura. Para facilitar la exposición, se hará referencia al modelo de aglomeración emanado de la corriente de la geografía económica como Cluster Económico Creativo (CEC) y al proveniente de los estudios urbanos, como Distrito Urbano Creativo (DUC).

Se denomina -estudios mixtos- a la literatura que mezcla el enfoque de CEC con el enfoque de DUC. Por ejemplo, Santagata (2002) parte de una definición de distrito 
industrial en la que enfatiza los componentes típicos de un CEC, las relaciones entre productores, la existencia de una atmosfera industrial, etc. Sin embargo, al presentar su tipología de distritos cae en una combinación de CEC y de DUC. Su primer tipo es lo que llama "distritos alrededor de un certificado de origen". Efectivamente, este tipo de producción alrededor de un producto protegido vía certificado de origen tiende a revelar un CEC, piénsese en productos agropecuarios procesados (Región de producción de vino la Ribera del Duero en España o la región de Tequila en México). Sin embargo, el resto de las categorías de Santagata no están justificadas con la definición de cluster económico y se vinculan directamente con el DUC. Santagata incorpora en su tipología el Distrito de Museo como una zona dentro de una ciudad con aglomeración de museos que genera atracción de artistas y de turismo. Otro tipo de tipología de distrito es la de Distritos Culturales Metropolitanos, definidos como la "aglomeración de edificios dedicados a las artes escénicas, museos y organizaciones que producen bienes y servicios culturales" (Santagata, 2002, p. 11).

La mezcla de los dos enfoques es aún más evidente y extendida en la arena de las políticas públicas de planeación urbana en donde el discurso de los clusters económicos ha sido usado para justificar proyectos urbanos a escala barrial. Bajo el argumento de crear empleo y detonar la formación de un cluster económico, se explica que las ventajas de la proximidad espacial entre empresas, instituciones y trabajadores generan innovación y mayor productividad. Un distrito urbano es, bajo esta óptica, el contenedor ideal para detonar un cluster económico debido a la extrema proximidad entre sus empresas y trabajadores (Homan, 2014).

La intervención en la escala intraurbana bajo la justificación de generar un CEC se expresa en políticas públicas que pueden incluso ser disruptivas para CECs en funcionamiento. De Propris y Wei (2007) analizan el caso del distrito joyero de Birmingham y muestra cómo el establecimiento de un proyecto de dinamizar la zona bajo un enfoque de distrito industrial en realidad impulsa la transformación de la zona en un centro de consumo de lujo, lo que debilita el entramado socioeconómico del histórico distrito joyero, ya que al elevarse los precios del suelo los talleres de joyería se ven obligados a abandonar la zona. La transformación de centros de producción creativa en centros de consumo se evidencia en la gran cantidad de políticas de desarrollo turístico que se basan en la promoción de distritos creativos o barrios de arte (Pappalepore, Maitland, \& Smith, 2014; Richards, 2011), que se reducen a zonas de consumo estetizado.

En esta línea, Pratt (2009) ha criticado que las políticas de germinación de distritos creativos han tenido resultados limitados en cuanto al desarrollo de clusters económicos. En los casos que ha estudiado en Inglaterra, el resultado de estas políticas ha sido la atracción de turistas, consumidores y residentes de alto ingreso y no la generación de un CEC. territarias 34

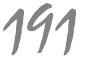


El principal problema con este tipo de políticas es que dejan fuera la lógica y complejidad de la producción en sectores creativos y se enfocan, casi exclusivamente, en la cuestión del consumo de productos, servicios y espacios públicos estetetizados. Existe una amplia literatura que ha documentado que estos proyectos terminan en procesos de gentrificación en los que los productores, artistas y empresas creativas pequeñas son expulsadas por el propio proceso de revalorización del suelo del distrito urbano, siendo los beneficiarios los desarrolladores y tenientes de bienes raíces (Brown, O'Connor \& Cohen, 2000; Chapple, Jackson \& Martin, 2010; Darchen, 2013; Hutton, 2010; Pratt, 2009; Scott, 2014; van Heur, 2009).

Se precisa que el argumento no cuestiona la formación de subcentros concentradores de empleo dentro de las ciudades. La literatura sobre estructura urbana que estudia este fenómeno lo ha documentado y explicado ampliamente (Alegría, 2009; McMillen, 2003). Se debe recordar que un subcentro urbano es principalmente una concentración de empresas y trabajadores. De aquí, que existe la posibilidad de que un cluster económico coincida espacialmente con un subcentro urbano. En el caso de ciudades monocéntricas, definitivamente esto es posible, pero cuando las ciudades pasan cierto tamaño generan subcentros volviéndose policéntricas y la relación entre cluster económico y subcentro se vuelve más problemática. ¿Es cada subcentro un cluster o un cluster se expresa en varios subcentros?
Se concluye esta sección afirmando que los DUC son distintos a los CEC. Los primeros denotan solamente la densidad de un tipo de actividad en una zona de la ciudad que le da cierto carácter distinguible de otras zonas y que afecta su proceso de transformación urbana (valor del suelo, medio ambiente construido, etc.). Los segundos son sistemas económicos y mercados laborales aglomerados, a diferencia de los anteriores, su escala territorial es la región o zona metropolitana. Es claro que hay una interrelación entre ambos tipos de distritos, sin embargo, este aspecto ha sido poco desarrollado en la literatura. Por el contrario, lo que se observa en ambos enfoques es una potencial transpolación de conceptos entre escalas geográficas sin su correspondiente adecuación. Por otra parte, los estudios de CEC y DUC tienden a no usar métodos de identificación de los clusters/distritos, partiendo de Clusters/ distritos conocidos, en esta ambigüedad de identificación a veces se incluyen distritos intraurbanos o ciudades enteras, lo que crea más confusión. En la práctica, esto ha dado cobijo teórico a proyectos inmobiliarios que en poco ayudan a la formación de clusters económicos. En el caso de México, se pueden citar dos ejemplos: el proyecto de las ZODEs (Zonas de Desarrollo Económico y Social) (Gobierno del Distrito Federal, 2015), propuestas por el Gobierno del Distrito Federal, que incluye la promoción de un "Corredor Creativo" sobre avenida Chapultepec o el proyecto llamado "Ciudad Creativa Digital", distrito designado en el centro de la ciudad de Guadalajara 
en el que el gobierno local pretende desarrollar un cluster creativo basado en sectores como "producción de cine, televisión, videojuegos, animación digital, medios interactivos, aplicaciones móviles, internet y otras industrias creativas" (Municipio de Guadalajara, 2014). En ambos proyectos se ve que se usa la retórica de las ventajas económicas de la proximidad espacial en una escala intraurbana que no corresponde con la escala de un CEC. El impacto de estos estos proyectos, de realizarse, tendrá una alta probabilidad de limitarse a un impacto inmobiliario.

\section{Identificación de distritos creativos en la Ciudad de México}

En esta sección se reporta la investigación descriptiva sobre la estructura espacial y características económicas de las industrias creativas en la Ciudad de México y su zona metropolitana. Primero, se evalúa el nivel de concentración de los sectores creativos en la Ciudad, de tal manera que permita hablar de la posible existencia de un cluster creativo. En segundo lugar, se analiza la distribución de las empresas creativas para identificar posibles DUC. Los Distritos son analizados en sus estructura económica interna buscando indicios de la formación de CEC diferenciados.

Para conducir al análisis se utilizaron los censos económicos 2009 y la base a datos de la DENUE 2012 que permiten la ubicación individual de las empresas en sectores clasificados en esta investigación como de carácter cultural/creativo. La selección de las actividades incluidas en el estudio se basa en el Sistema de Clasificación Industrial de América del Norte (SCIAN), que se utiliza en los censos económicos de México. La selección de actividades se acotó a las directamente relacionadas con actividades artísticas o con actividades cuyo principal producto/servicio tuviese un contenido simbólico o cultural significativo. Las áreas que se cubren son (a) la industria editorial, la industria de audio y la audiovisual, (b) el área de diseño y arquitectura, (c) la publicidad, (d) el sector educativo y comercial vinculado al arte y (e) artistas, compañías artísticas y sus representantes. La composición por subclase se puede ver en la tabla 1. Esta clasificación corresponde con la propuesta por la UNESCO (UNESCO y UNDP, 2010).

\subsection{Importancia de los sectores creativos en el ZMVM}

A partir de la clasificación anterior, se analizaron los censos económicos 2009 (últimos disponibles a la fecha) para evaluar el grado de concentración de los sectores creativos en la ciudad de México. En la tabla 2 se puede observar para cada clase o subsector seleccionado el peso nacional que tiene el ZMVM en cuanto a unidades económicas, empleo, remuneraciones y valor agregado censal bruto. La última columna presenta el "coeficiente de localización" (QL), que relaciona la proporción del empleo que cada clase o subsector representa a nivel nacional y la proporción que el empleo de cada clase o subsector tiene en el Distrito 
Tabla 1. Definición estadística de los sectores creativos 


\begin{tabular}{|l|l|l|l|l|}
\hline & $\begin{array}{l}\text { Edición y difusión de } \\
\text { contenido exclusivamente } \\
\text { a través de Internet y } \\
\text { servicios de búsqueda en } \\
\text { la red 519130 }\end{array}$ & $\begin{array}{l}\text { Agencias de compra de } \\
\text { medios a petición del } \\
\text { cliente 541830 }\end{array}$ & & \\
\hline & $\begin{array}{l}\text { Agencias de } \\
\text { representación de } \\
\text { medios 541840 }\end{array}$ & $\begin{array}{l}\text { Agencias de anuncios } \\
\text { publicitarios 541850 }\end{array}$ & & \\
\hline & $\begin{array}{l}\text { Servicios de } \\
\text { investigación de } \\
\text { mercados y encuestas de } \\
\text { opinión pública 541910 }\end{array}$ & & \\
\hline & & & \\
\hline
\end{tabular}

Fuente: elaboración propia con base en el Sistema de Clasificación Industrial de América del Norte SCIAN 2007.

Tabla 2. Sectores creativos en el ZMVM y su importancia nacional

\begin{tabular}{|c|c|c|c|c|c|c|}
\hline SCIAN & Nombre & $\begin{array}{l}\text { Unidades } \\
\text { Económi- } \\
\text { cas \% del } \\
\text { Nacional }\end{array}$ & $\begin{array}{l}\text { Personal } \\
\text { Ocupado } \\
\% \text { del Na- } \\
\text { cional }\end{array}$ & $\begin{array}{l}\text { Total de } \\
\text { remunera- } \\
\text { ciones \% del } \\
\text { Nacional }\end{array}$ & $\begin{array}{c}\text { Valor agre- } \\
\text { gado censal } \\
\text { Bruto \% del } \\
\text { Nacional }\end{array}$ & $\begin{array}{l}\text { Coefi- } \\
\text { ciente de } \\
\text { Localiza- } \\
\text { ción }\end{array}$ \\
\hline 466313 & $\begin{array}{l}\text { Comercio al por menor de } \\
\text { antigüedades y obras de arte }\end{array}$ & 23,3 & 28,8 & 40,6 & 55,5 & 1,2 \\
\hline 5111 & $\begin{array}{l}\text { Edición de periódicos, revistas, libros } \\
\text { y similares, y edición de estas publica- } \\
\text { ciones integradas con la impresión }\end{array}$ & 17,1 & 38,3 & 50,4 & 65,5 & 1,6 \\
\hline 5121 & Industria fílmica y del video & 24,4 & 47,0 & 57,6 & 96,9 & 2,0 \\
\hline 5122 & Industria del sonido & 24,9 & 72,3 & 95,2 & 89,4 & 3,1 \\
\hline 51913 & $\begin{array}{l}\text { Edición y difusión de contenido } \\
\text { exclusivamente a través de Internet } \\
\text { y servicios de búsqueda en la red }\end{array}$ & * & 79,6 & 98,0 & 99,9 & 3,4 \\
\hline 54131 & Servicios de arquitectura & 22,0 & 37,3 & 52,8 & 41,4 & 1,6 \\
\hline 54132 & $\begin{array}{l}\text { Servicios de arquitectura de paisaje } \\
\text { y urbanismo }\end{array}$ & 10,2 & 41,0 & 52,0 & 70,8 & 1,7 \\
\hline 54134 & Servicios de dibujo & 17,8 & 26,8 & 30,6 & 29,8 & 1,1 \\
\hline 54141 & Diseño y decoración de interiores & 18,7 & 31,0 & 38,5 & 46,7 & 1,3 \\
\hline
\end{tabular}




\begin{tabular}{|c|c|c|c|c|c|c|}
\hline SCIAN & Nombre & $\begin{array}{l}\text { Unidades } \\
\text { Económi- } \\
\text { cas \% del } \\
\text { Nacional }\end{array}$ & $\begin{array}{l}\text { Personal } \\
\text { Ocupado } \\
\% \text { del Na- } \\
\text { cional }\end{array}$ & $\begin{array}{l}\text { Total de } \\
\text { remunera- } \\
\text { ciones \% del } \\
\text { Nacional }\end{array}$ & $\begin{array}{l}\text { Valor agre- } \\
\text { gado censal } \\
\text { Bruto \% del } \\
\text { Nacional }\end{array}$ & $\begin{array}{l}\text { Coefi- } \\
\text { ciente de } \\
\text { Localiza- } \\
\text { ción }\end{array}$ \\
\hline 54142 & Diseño industrial & 18,5 & 35,2 & 27,9 & 39,3 & 1,5 \\
\hline 54143 & Diseño gráfico & 27,0 & 51,8 & 64,2 & 57,4 & 2,2 \\
\hline 54149 & $\begin{array}{l}\text { Diseño de modas y otros diseños } \\
\text { especializados }\end{array}$ & 10,0 & 49,9 & 76,5 & 71,6 & 2,1 \\
\hline 54181 & Agencias de publicidad & 25,6 & 59,2 & 73,5 & 66,9 & 2,5 \\
\hline 54182 & Agencias de relaciones públicas & 44,8 & 93,2 & 97,3 & 96,4 & 4,0 \\
\hline 54183 & $\begin{array}{l}\text { Agencias de compra de medios a } \\
\text { petición del cliente }\end{array}$ & 12,5 & 51,5 & 63,3 & 80,6 & 2,2 \\
\hline 54184 & Agencias de representación de medios & 157 & 49,9 & 77,9 & 42,7 & 2,1 \\
\hline 54185 & Agencias de anuncios publicitarios & 18,8 & 42,1 & 61,6 & 63,4 & 1,8 \\
\hline 54191 & $\begin{array}{l}\text { Servicios de investigación de mercados } \\
\text { y encuestas de opinión pública }\end{array}$ & 55,0 & 69,9 & 89,0 & 81,7 & 3,0 \\
\hline 61161 & Escuelas de arte & 24,3 & 28,2 & 35,0 & 33,1 & 1,2 \\
\hline 71111 & Compañías de teatro & 22,2 & 63,6 & 94,6 & 94,8 & 2,7 \\
\hline 71112 & Compañías de danza & * & 28,8 & 0,0 & 0,6 & 1,2 \\
\hline 71113 & Cantantes y grupos musicales & 1,5 & 7,6 & 7,4 & 8,6 & 0,3 \\
\hline 71119 & $\begin{array}{l}\text { Otras compañías y grupos de } \\
\text { espectáculos artísticos }\end{array}$ & 2,0 & 32,8 & 35,4 & 70,8 & 1,4 \\
\hline 71132 & $\begin{array}{l}\text { Promotores de espectáculos } \\
\text { artísticos, culturales, deportivos } \\
\text { y similares que no cuentan con } \\
\text { instalaciones para presentarlos }\end{array}$ & 12,6 & 24,1 & 31,6 & 41,0 & 1,0 \\
\hline 71141 & $\begin{array}{l}\text { Agentes y representantes de artistas, } \\
\text { deportistas y similares }\end{array}$ & 16,7 & 46,1 & 38,2 & 40,0 & 2,0 \\
\hline \multirow[t]{2}{*}{71151} & $\begin{array}{l}\text { Artistas, escritores y técnicos } \\
\text { independientes }\end{array}$ & 8,7 & 34,1 & 35,7 & 38,9 & 1,4 \\
\hline & Total ZMVM del Nacional & 20,7 & 45,3 & 62,7 & 65,4 & 1,9 \\
\hline
\end{tabular}

* Datos omitidos por el INEGI debido al principio de confidencialidad

Fuente: Elaboración propia con datos de los Censos Económicos 2009, INEGI. 
Federal. Si la proporción de empleo es igual en el nivel nacional y el local, el coeficiente es de 1. Las cifras mayores a uno indican especialización, ya que la proporción local es mayor que la nacional.

Tomando en conjunto todos los sectores seleccionados, el peso del ZMVM con respecto al nacional es muy grande, tiene el $20,7 \%$ de las empresas creativas y el $45,3 \%$ del empleo y un coeficiente de localización de 1,9. Aún más importante es el desempeño económico; el ZMVM genera el 62,7\% de las remuneraciones, el 65,4\% del valor agregado censal bruto. A nivel sector hay varios aspectos que son importantes. En primer lugar, las actividades alrededor de la publicidad (investigación, conceptualización, producción, coordinación) que se localizan en el ZMVM lideran claramente a nivel nacional (en promedio constituyen el $29 \%$ de las empresas, $61 \%$ del empleo, $72 \%$ del valor agregado nacional). En segundo lugar, destacan las actividades de cine y video que ocupan a 5 trabajadores de cada 10 en el país, y las de la industria del sonido que emplean a 7 de cada 10 a nivel nacional. El valor agregado de estas dos actividades es aún más elevado, alcanza niveles del $96,9 \%$ y $89,4 \%$ del valor agregado nacional. En tercer lugar, están las actividades vinculadas con el diseño y la arquitectura, de las cuales se destacan el diseño de modas ( $50 \%$ de los empleos y 71,6\% del valor agregado nacional), los servicios de arquitectura del paisaje y urbanismo (41\% del empleo y $70,8 \%$ del valor agregado nacional) y el diseño gráfico $(51,8 \% \mathrm{del}$ empleo y el $57,4 \%$ del valor agregado). En cuarto lugar, el ZMVM se destaca también en sectores creativos tradicionales; las compañías de teatro ocupan al 63,6\% del empleo y presenta un Coeficiente de localización (QL) de 2,7.

Resumiendo, la Ciudad de México concentra segmentos muy importantes de varios de los sectores seleccionados. Esto comprueba que, tal como sucede en otros países, las empresas creativas tienden a concentrarse en las ciudades más grandes de cada país (Florida, Mellander \& Stolarick, 2010; Florida, Mellander \& Stolarick, 2011). Por otra parte, los altísimos niveles de especialización en las actividades de publicidad son muy relevantes, ya que, como han tratado otros textos, la publicidad es un nodo en el que muchas actividades creativas se vinculan entre sí y constituyen un cluster en sí mismo (Mercado, 2011). Se puede concluir que la información presentada señala la existencia plausible de un cluster/ distrito creativo en la ciudad de México.

\subsection{Distribución espacial de las actividades creativas en la Zona Metropolitana del Valle de México}

Una vez que se ha documentado la existencia de una aglomeración relevante de empresas creativas en la ciudad de México, se analiza la distribución espacial de estas empresas dentro de la zona metropolitana para verificar si se han formado distritos internos. Se empleará el método de identificación a partir de densidad de actividades 
4 Colonias de acuerdo con la cartografía de navteq. que es usado en la literatura de DUC. Para el análisis de la distribución espacial se utilizó la base de datos del INEGI DENUE 2012.

Para la identificación de las colonias con concentración significativa de empresas, se hicieron dos cohortes, la primero fijada por la media, más una desviación estándar (9 empresas) y una segunda cohorte a partir de la media, más dos desviaciones estándar (16 empresas). Entre ambas cohortes se identificaron 114 colonias con concentraciones significativas que representan el $2 \%$ del total de las 5623 colonias en la zona metropolitana ${ }^{4}$ (tabla 3 ), en conjunto, estás colonias contienen el 55,4\% del total de empresas en sectores creativos del ZMVM. La figura 1 presenta el conjunto de colonias con concentraciones por encima de una desviación estándar de empresas en los sectores seleccionados. La imagen

Tabla 3. Colonias con mayor concentración (una desviación estándar)

\begin{tabular}{|c|c|c|c|c|c|}
\hline \multicolumn{2}{|r|}{ D.F. } & \multicolumn{2}{|c|}{ D.F. } & \multicolumn{2}{|c|}{ Edo de México } \\
\hline Delegación & Colonia & Delegación & Colonia & Municipio & Colonia \\
\hline \multirow{12}{*}{$\begin{array}{l}\text { Benito } \\
\text { Juárez }\end{array}$} & Narvarte Poniente & Cuahutemoc & Centro & Chalco & Benito Juárez \\
\hline & Narvarte Oriente & & Tabacalera & $\begin{array}{c}\text { Coacalco de } \\
\text { Berriozbal }\end{array}$ & El Laurel \\
\hline & Del Valle Centro & & Hipódromo & $\begin{array}{c}\text { Ecatepec de } \\
\text { Morelos }\end{array}$ & Hogares Marla \\
\hline & Insurgentes San Borja & & Condesa & & Izcalli Ecatepec \\
\hline & Del Valle & & $\begin{array}{l}\text { Hipódromo de la } \\
\text { Condesa }\end{array}$ & & $\begin{array}{c}\text { Jardines de } \\
\text { Morelos }\end{array}$ \\
\hline & Del Valle Sur & & Morelos & & $\begin{array}{l}\text { Jardines de Santa } \\
\text { Clara }\end{array}$ \\
\hline & Tlacoquemecatl & & Guerrero & Ixtapaluca & Ixtapaluca Centro \\
\hline & Acacias & & Buenavista & $\begin{array}{l}\text { Naucalpan de } \\
\text { Jurez }\end{array}$ & Ciudad Satelite \\
\hline & Portales Sur & & $\begin{array}{l}\text { Santa Maria La } \\
\text { Ribera }\end{array}$ & & Los Álamos \\
\hline & Portales Norte & & San Rafael & & $\begin{array}{l}\text { Bosques de } \\
\text { Echegaray }\end{array}$ \\
\hline & Santa Cruz Atoyac & & Cuauhtemoc & & $\begin{array}{l}\text { Fracc Industrial } \\
\text { Alce Blanco }\end{array}$ \\
\hline & $\begin{array}{l}\text { Gral. Pedro Maria } \\
\text { Anaya }\end{array}$ & & Juárez & & El Parque \\
\hline
\end{tabular}

Continúa 


\begin{tabular}{|c|c|c|c|c|c|}
\hline \multicolumn{2}{|r|}{ D.F. } & \multicolumn{2}{|r|}{ D.F. } & \multicolumn{2}{|c|}{ Edo de México } \\
\hline Delegación & Colonia & Delegación & Colonia & Municipio & Colonia \\
\hline \multirow{17}{*}{$\begin{array}{l}\text { Benito } \\
\text { Juárez }\end{array}$} & Álamos & & Roma Norte & Nezahualcyotl & Benito Juárez \\
\hline & Moderna & & Doctores & & $\begin{array}{c}\text { Campestre } \\
\text { Guadalupana }\end{array}$ \\
\hline & Vertiz Narvarte & & Roma Sur & & $\begin{array}{c}\text { Bosques de } \\
\text { Aragón }\end{array}$ \\
\hline & Independencia & & Obrera & & Estado de México \\
\hline & Letran Valle & & Algarin & Texcoco & Centro Pte \\
\hline & San Simón Ticumac & $\begin{array}{c}\text { Alvaro } \\
\text { Obregón }\end{array}$ & San Ángel & $\begin{array}{c}\text { Tlalnepantla de } \\
\text { Baz }\end{array}$ & $\begin{array}{c}\text { Tlanepantla de Baz } \\
\text { Centro }\end{array}$ \\
\hline & Nonoalco & & Los Alpes & & Valle Dorado \\
\hline & $\begin{array}{l}\text { Ciudad de los } \\
\text { Deportes }\end{array}$ & & Guadalupe Inn & & La Romana \\
\hline & Noche Buena & & Florida & & $\begin{array}{l}\text { San Lucas } \\
\text { Tepetlacalco }\end{array}$ \\
\hline & San Juan & & La Otra Banda & & Viveros del Valle \\
\hline & $\begin{array}{l}\text { Extremadura } \\
\text { Insurgentes }\end{array}$ & & Merced Gómez & $\begin{array}{c}\text { Valle de Chalco } \\
\text { Solidaridad }\end{array}$ & Jardin \\
\hline & $\begin{array}{c}\text { San Pedro de los } \\
\text { Pinos }\end{array}$ & & $\begin{array}{l}\text { Jardines del } \\
\text { Pedregal }\end{array}$ & Zumpango & Barrio San Juan \\
\hline & Ampl Napoles & Coyoacán & Villa Coyoacán & & \\
\hline & Nápoles & & Parque San Andrés & & \\
\hline & San José Insurgentes & & Del Carmen & & \\
\hline & Insurgentes Mixcoac & & $\begin{array}{l}\text { Campestre } \\
\text { Churubusco }\end{array}$ & & \\
\hline & Crédito Constructor & & Ajusco & & \\
\hline \multirow{3}{*}{$\begin{array}{l}\text { Miguel } \\
\text { Hidalgo }\end{array}$} & Lomas Altas & & $\begin{array}{c}\text { Pedregal de Santo } \\
\text { Domingo }\end{array}$ & & \\
\hline & $\begin{array}{l}\text { Lomas de } \\
\text { Chapultepec }\end{array}$ & & Fracc. Res Cafetales & & \\
\hline & Molino del Rey & Tlalpan & Tlalpan Centro 1 & & \\
\hline
\end{tabular}




\begin{tabular}{|c|c|c|c|c|c|}
\hline \multicolumn{2}{|r|}{ D.F. } & \multicolumn{2}{|c|}{ D.F. } & \multicolumn{2}{|c|}{ Edo de México } \\
\hline Delegación & Colonia & Delegación & Colonia & Municipio & Colonia \\
\hline \multirow{20}{*}{$\begin{array}{l}\text { Miguel } \\
\text { Hidalgo }\end{array}$} & $\begin{array}{l}\text { 2da. Secc. del Bosque } \\
\text { de Chapultepec }\end{array}$ & & $\begin{array}{l}\text { Jardines en la } \\
\text { Montaa }\end{array}$ & & \\
\hline & Verónica Anzures & $\begin{array}{l}\text { Venustiano } \\
\text { Carranza }\end{array}$ & Jardín Balbuena & & \\
\hline & Anahuac & $\begin{array}{l}\text { Cuajimalpa } \\
\text { de Morelos }\end{array}$ & $\begin{array}{c}\text { Centro Comercial } \\
\text { Santa Fe }\end{array}$ & & \\
\hline & Casa Blanca & $\begin{array}{l}\text { Gustavo A. } \\
\text { Madero }\end{array}$ & Lindavista & & \\
\hline & Tacuba & Iztacalco & Agricola Oriental & & \\
\hline & Irrigación & Iztapalapa & Escuadrón 201 & & \\
\hline & Los Morales & & & & \\
\hline & Granada & & & & \\
\hline & $\begin{array}{c}\text { Los Morales Secc } \\
\text { Palmas }\end{array}$ & & & & \\
\hline & Polanco Reforma & & & & \\
\hline & Palmitas & & & & \\
\hline & Polanco Chapultepec & & & & \\
\hline & $\begin{array}{l}\text { Bosques de } \\
\text { Chapultepec }\end{array}$ & & & & \\
\hline & Chapultepec Morales & & & & \\
\hline & Anzures & & & & \\
\hline & Nueva Anzures & & & & \\
\hline & Reforma Social & & & & \\
\hline & Bosques de las Lomas & & & & \\
\hline & Escandón & & & & \\
\hline & $\begin{array}{l}\text { San Miguel } \\
\text { Chapultepec }\end{array}$ & & & & \\
\hline
\end{tabular}

\section{territarios 34}

Fuente: elaboración propia. 
muestra claramente el dominio de tres delegaciones centrales en la concentración de las industrias creativas: Cuauhtémoc, Benito Juárez y Miguel Hidalgo, en la frontera con Benito Juárez se añaden las delegaciones de Coyoacán y Álvaro Obregón en la sección de San Ángel. Fuera de esta extensa aglomeración se destacan algunas zonas de la Delegación Iztacalco, Venustiano Carranza y Gustavo A. Madero en el D.F. y Naucalpan, Tlalnepantla, Ecatepec, Netzahualcóyotl y Texcoco en el Estado de México.
El patrón de distribución que aparece en la figura 1 muestra un gran distrito central y un conjunto de concentraciones significativas de empresas en colonias aisladas. Esta distribución plantea algunos interrogantes. En primer lugar, solo las colonias insulares de alta concentración presentan una escala geográfica coherente con la que se maneja en el enfoque de DUC. En segundo lugar, el gran distrito central no coincide con la escala geográfica de los DUC, ya que un espacio tan grande y diverso en cuanto a niveles de ingreso, medio ambiente cons-

Figura 1. Амvм Concentración de Empresas Sectores Creativos

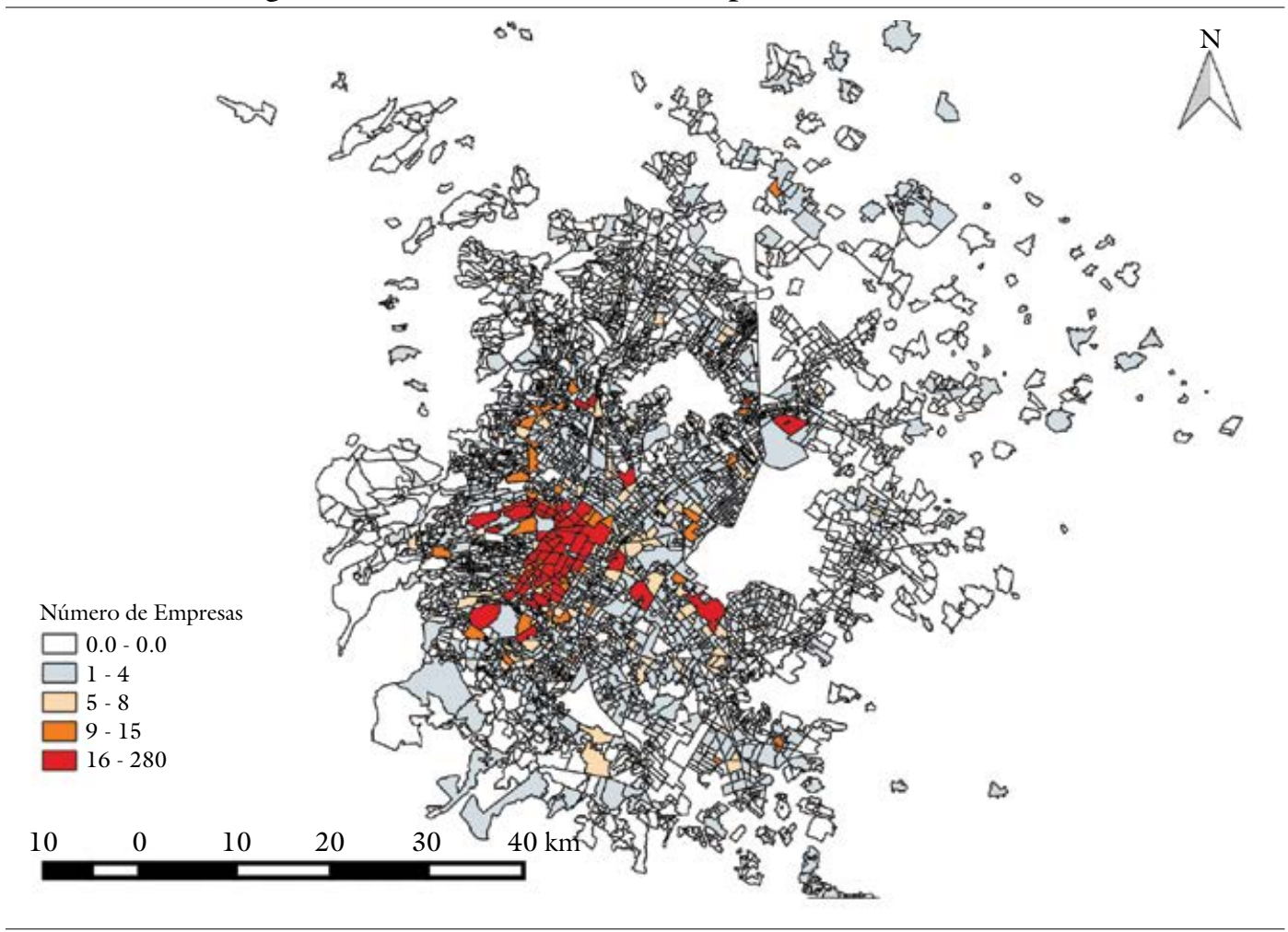

Fuente: elaboración propia a partir de DENUE 2012. 
${ }^{5}$ Ver trabajo de Connolly (2005) sobre los diversos tipos de poblamiento su impacto en la imagen y estructura urbana de la metrópoli.

territarias 34 truido y tipo de poblamiento ${ }^{5}$ no podría constituir un mismo tipo de perfil urbano. Sin embargo, para este último caso, cabe la posibilidad de que, aunque existe una contigüidad física entre las colonias de mayor densidad, en realidad sean distritos urbanos diferenciados. Con el objetivo de verificar la existencia de áreas con rasgos económicos diferenciables en una escala acorde con los DUC, se han dividido las zonas de alta densidad en distritos a partir de los criterios que se delinean a continuación.

Para formar los distritos, se atendieron los siguientes criterios: (a) se seleccionaron las colonias con más de dos desviaciones estándar (16 empresas), (b) estas colonias se agregaron en conjuntos/distritos, ya sea porque tienen contigüidad física o porque están cercanamente conectadas por una vialidad principal, (c) se agregaron a los anteriores distritos las colonias con una densidad de más de una desviación estándar (9 empresas) y con contigüidad física y (d) en el caso de la zona central, para dividirla en distritos más pequeños se utiliza el criterio subjetivo del 'carácter urbano' que tiene cada zona dentro de una ciudad y que aparece en la literatura de DUC que presentamos en la primera sección.

Utilizando solamente los tres primeros criterios se identificaron los distritos (D7) Corredor Satélite, (D8) Ecatepec, (D9) Jardín Balbuena, (D10) Agrícola Oriental, (D11) Netzahualcóyotl, (D12) Lindavista y (D13) Chalco. Para dividir la gran concentración central identificada con los tres primeros criterios se añadió el criterio subjetivo de 'carácter urbano' resultando los distritos (D1) Coyoacán San Ángel, (D2) del Valle, (D3) Roma-Condesa, (D4) Polanco-Santa Fe, (D5) Centro, (D6) Santa María la Ribera (figura 2). El perfil de carácter urbano de estos últimos distritos es el siguiente: La Roma-Condesa con un atractivo visual conformado por parques, jardines y camellones, predominio de casas y edificios bajos, y una estética que combina la arquitectura de los años veinte a los cuarenta con las nuevas construcciones posmodernas a partir de los noventa. La zona Coyoacán-San Ángel, marcada por la presencia de grandes instituciones culturales y educativas, con un carácter urbano que la conecta más a un suburbio de la ciudad con una estética colonial de grandes casonas y plazas cívicas. El Distrito del Valle, una zona que se densifica a partir de los años cincuenta y sesenta con un carácter residencial de clase media alta, en donde se combinan edificios de departamentos con casas de gran tamaño, cruzada por el principal eje comercial de la ciudad, la avenida insurgentes. El carácter urbano de esta zona es de un espacio moderno, de grandes avenidas sin muchos espacios públicos. Santa María la Ribera, barrio popular con cierto nivel de deterioro urbano pero con atractivas construcciones porfirianas, así como parques y jardines. Ciudad Satélite, típico suburbio de la segunda mitad del siglo Xx, uso predominantemente residencial unifamiliar, predominio del automóvil como forma de movilidad interna. Polanco-Las Lomas, el símbolo de las clases adineradas en la época posrevolucionaria de la ciudad de México, contexto urbano de la mejor infraestruc- 
tura urbana, dominada por lujosas casas de gran tamaño, con una transformación hacia edificios de altura, tanto de departamentos de lujo como de oficinas y hoteles. Y el Centro Histórico, símbolo de atractivo turístico, herencia arquitectónica que va de lo prehispánico hasta lo moderno y sus áreas circundantes con un perfil de clase trabajadora y alta concentración de pequeño comercio. En resumen, estos últimos distritos, más los insulares previamente identificados suman 13 distritos: (Dl) Coyoacán San Ángel, (D2) Del Valle, (D3) Roma-
Condesa, (D4) Polanco-Santa Fe, (D5) Centro, (D6) Santa María la Ribera, (D7) Corredor Satélite, (D8) Ecatepec, (D9) Jardín Balbuena, (D10) Agrícola Oriental, (D11) Netzahualcóyotl, (D12) Lindavista y (Dl3) Chalco (figura 2).

Una cuestión central que puede ayudar a discernir si tanto los DUC identificados son también CEC, es si cada uno muestra un perfil diferente en la actividad económica. Para observar esto, se analizan tres características; el tamaño promedio de las empresas, la especialización económica de cada distrito

Figura 2. Posibles Distritos Creativos en el AMvM

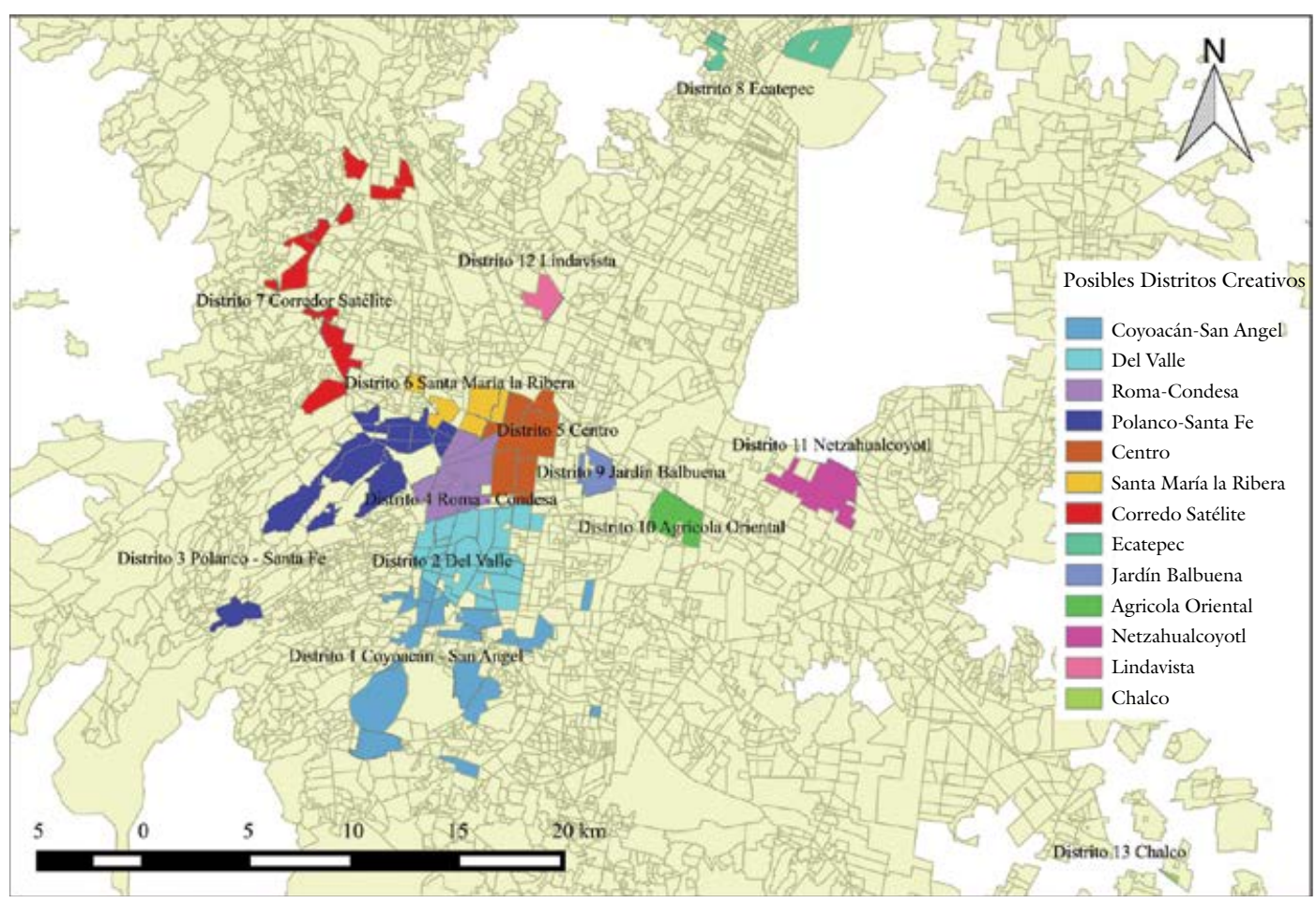

Fuente: elaboración propia. Distritación a partir de densidad de empresas, obtenido de la DENUE 2012 y descripción del carácter urbano a partir de observaciones directas. 
6 INEGI clasifica a las empresas con menos de 10 empleados como microempresas.

territarias 34 y el peso interno de cada sector creativo. Si los distritos muestran especializaciones, estructuras y predominio de tamaños de empresas distintos, esto indicaría la posibilidad de que cada uno fuera un CEC. Los resultados son los siguientes.

Los datos del número de trabajadores promedio por empresa en los 13 distritos muestran un dominio en todos ellos de microempresas ${ }^{6}$; el rango va de 2,5 empleados por empresa en el Distrito de Chalco a 10,7 en el Distrito de Polanco-Santa Fe. Dentro de esta homogeneidad, se puede distinguir un subconjunto de distritos que están por debajo de 3 empleados por empresa, todos ellos en la zona oriente de la ciudad (Agrícola Oriental, Chalco, Ecatepec y Netzahualcóyotl). Fuera de este grupo, las diferencias del tamaño de las empresas de acuerdo con el carácter urbano entre los distritos no son significativas. Por ejemplo, el Distrito Polanco-Santa Fe, donde se esperaba encontrar las empresas más grandes del ZMVM tiene en realidad el mismo promedio de empleados por empresa que el Distrito del Valle, Coyoacán San Ángel y Jardín Balbuena (9,7 a 10,7). Por otra parte, si bien el Distrito Centro tiene en promedio empresas muy pequeñas $(3,8)$ son muy parecidas a las del Corredor Satélite $(4,1)$.

En cuanto al perfil económico en cada distrito, se tomaron en cuenta tres elementos para evaluar qué tan diferentes o similares son: (a) la diversidad de sectores presentes en cada distrito, (b) la especialización económica y (c) los 5 sectores económicos más importantes de cada distrito.
La diversidad económica se calculó con un índice en el que el máximo posible de sectores en un distrito es de 34 , en el caso de que un distrito tuviera todos los sectores su índice sería de 1 , entre más alejado del 1 menos diversidad tiene y viceversa. Lo que se encuentra es que los distritos del 1 al 5 (Coyoacán-San Ángel, Del Valle, RomaCondesa, Polanco-Santa Fe, Centro) tienen un índice alto de diversidad sectorial, ubicándose entre 0,91 y 0,97. Después hay una categoría intermedia con un índice de 0,62 y 0,65 de los distritos 6 y 7 (Santa María la Ribera y Corredor Satélite); finalmente, los distritos con una diversidad baja en su composición sectorial son los distritos del 8 al 12 con un índice que va del 0,21 al 0,32 (Ecatepec, Jardín Balbuena, Agrícola Oriental, Netzahualcóyotl, Lindavista). Chalco, con sólo dos sectores representa un caso extremo, ya que tiene un índice de 0,06 . A este nivel, la información no sustenta la existencia de distritos diferenciados dentro de la zona central. En todo caso, indica una división entre distritos centrales y periféricos. Esta diferencia entre los Distritos Centrales (D1 a D5) y los periféricos (D8 a D13) se explorará con más detalle para ver si se sostiene está diferencia. Se hará referencia a la primera como Zona Central y a la segunda Zona Periférica. Se agregará una Zona Intermedia compuesta por los distritos 6 y 7, que también presentan similitud en su índice de diversidad.

Para analizar la especialización económica de los distritos se utilizó un coeficiente de localización (QL) calculado con base en la proporción del empleo en cada 
sector para cada distrito dividido por la misma proporción a nivel metropolitano, lo que da un índice en el que los sectores que obtienen más de 1 , tienen un grado de concentración de empleo mayor al que proporcionalmente presenta el conjunto de la zona metropolitana. Una vez calculado, se identificó si los QL > 1 pertenecen a distritos distintos o se comparten entre distritos. Si los sectores con QL mayores a 1 están en distritos diferentes son sectores únicos, esto indicaría que son distritos diferenciados; por el contrario, si los sectores con un QL mayor a 1 se comparten entre varios distritos, esto apuntaría a que son distritos similares. Además de lo anterior, se agregaron los distritos en las tres zonas (Central, Intermedia, Periférica) que se identificaron con el índice de diversidad para probar si a esta escala geográfica hay una diferenciación más importante que a nivel distrito.

Los resultados se muestran en Cuadro IV, lo que se debe destacar es que, todos los distritos presentan sectores mayores a 1 , lo que indica que efectivamente las aglomeraciones de empresas en estas áreas son significativas. En este sentido, también es relevante que todos los distritos presenten el $50 \%$ de sus sectores económicos creativos con un QL mayor a uno. Los datos arrojan que las estructuras económicas de los distritos tienen un alto nivel de similitud. En conjunto los distritos comparten al menos 3 sectores con un QLs de más de 1 , y 11 distritos de los 13, comparten $7 \mathrm{sec}$ tores con más de 1 QL. Otra forma de verlo es que con la excepción de tres Distritos
(D4, D5 y D9) los 10 restantes Distritos comparten todos sus sectores, es decir no tienen sectores "únicos".

Los que sí presentaron sectores únicos son El D4 Polanco-Santa Fe, el único con un QL mayor a $1(4,4)$ en el sector Agencias de compra de medios a petición del cliente (SCIAN 541830); El Distrito 5 Centro, donde el sector Compañías de Danza (SCIAN 711121) se concentra (QL 23.6). El caso del sector Artistas, actores y técnicos independientes (SCIAN 711510) solamente presenta un QL mayor a uno $(2,6)$ en el D9 Jardín Balbuena. El que estos tres distritos cuenten con sectores "únicos" es importante en su caracterización como CEC, pero hay que hacer notar que el $\mathrm{D} 4$, además del sector único, comparte 12 sectores con QL $>1$ con al menos otro distrito, el D5 lo hace con 9 sectores y el D9 con 4 (tabla 4 ).

Sí se agrupan los distritos a partir de la clasificación que emergió con el índice de diversidad, se pueden encontrar datos de una diferencia más significativa a esta escala espacial. La Zona Central (Dl al 5 ) cuenta con 9 sectores únicos con un $\mathrm{QL}>=1$, y la Zona Periférica presenta 3 sectores únicos. Estos datos indican que estas dos Zonas tienen diferencias de mayor peso dentro de la ciudad. La zona intermedia (Distritos 6 y 7 ), sin embargo, no presenta ningún sector único y los que tiene con un $\mathrm{QL}>=1$ son compartidos con la Zona Central, por lo que se podrían agregar a esta zona, ya que no tienen ninguna diferencia. Es también interesante que la Zona Centro y la Periferia comparten 15 sectores, por lo que hay rasgos de diferenciación y de similitud. territarios 34 205 
Tabla 4. Estructura Económica de los Distritos con base a Coeficientes de Localización

\begin{tabular}{|c|c|c|c|c|c|c|c|c|c|c|c|c|c|c|c|c|c|}
\hline & $\begin{array}{l}\text { Coyoa- } \\
\text { cán - San }\end{array}$ & $\begin{array}{c}\text { Del } \\
\text { Valle }\end{array}$ & $\begin{array}{l}\text { Roma } \\
\text { - Con- }\end{array}$ & $\begin{array}{c}\text { Polanco } \\
\text { - Santa } \\
\text { Fe }\end{array}$ & Centro & $\begin{array}{c}\text { Santa } \\
\text { María la }\end{array}$ & $\begin{array}{l}\text { Cor- } \\
\text { redor }\end{array}$ & $\begin{array}{c}\text { Ecate- } \\
\text { pec }\end{array}$ & $\begin{array}{l}\text { Jardín } \\
\text { Balbue- }\end{array}$ & $\begin{array}{l}\text { Agrícola } \\
\text { Oriental }\end{array}$ & Netza- & $\begin{array}{c}\text { Linda- } \\
\text { vista }\end{array}$ & Chalco & & \multicolumn{3}{|c|}{ Agregados tres zonas } \\
\hline ZMVM & Distrito 1 & $\begin{array}{c}\text { Distri- } \\
\text { to } 2\end{array}$ & $\begin{array}{c}\text { Distri- } \\
\text { to } 3\end{array}$ & $\begin{array}{c}\text { Distri- } \\
\text { to } 4\end{array}$ & $\begin{array}{l}\text { Distri- } \\
\text { to } 5\end{array}$ & $\begin{array}{c}\text { Distri- } \\
\text { to } 6\end{array}$ & $\begin{array}{c}\text { Distri- } \\
\text { to } 7\end{array}$ & $\begin{array}{c}\text { Distri- } \\
\text { to } 8\end{array}$ & $\begin{array}{c}\text { Distri- } \\
\text { to } 9\end{array}$ & $\begin{array}{c}\text { Distrito } \\
10\end{array}$ & $\begin{array}{c}\text { Distrito } \\
11\end{array}$ & $\begin{array}{c}\text { Distrito } \\
12\end{array}$ & $\begin{array}{c}\text { Distrito } \\
13\end{array}$ & & $\begin{array}{c}\text { Zona } \\
\text { central }\end{array}$ & $\begin{array}{l}\text { Inter- } \\
\text { media }\end{array}$ & $\begin{array}{c}\text { Perifé- } \\
\text { rica }\end{array}$ \\
\hline SCIAN & Q Loc* & Q Loc & Q Loc & Q Loc & Q Loc & Q Loc & Q Loc & Q Loc & Q Loc & Q Loc & Q Loc & Q Loc & Q Loc & $\begin{array}{l}\text { \# Q- } \\
\text {-Loc }\end{array}$ & & & \\
\hline 466313 & 0,31 & 0,27 & 1,84 & 0,76 & 3,92 & 0,13 & 5,26 & 0,00 & 0,00 & 0,00 & 2,65 & 0,00 & 0,00 & 4 & 1 & 1 & 1 \\
\hline 511111 & 0,00 & 0,30 & 0,30 & 0,09 & 10,04 & 0,00 & 20,16 & 0,00 & 0,00 & 0,00 & 0,00 & 0,00 & 0,00 & 2 & 1 & 1 & \\
\hline 511112 & 0,00 & 1,80 & 0,43 & 0,09 & 4,30 & 5,03 & 0,21 & 0,48 & 0,00 & 0,00 & 2,92 & 0,00 & 0,00 & 4 & 1 & 1 & 1 \\
\hline 511131 & 0,07 & 0,39 & 0,82 & 0,46 & 3,39 & 10,06 & 3,17 & 0,00 & 0,00 & 0,00 & 0,00 & 0,00 & 0,00 & 3 & 1 & 1 & \\
\hline 512111 & 0,32 & 1,16 & 1,00 & 0,99 & 0,34 & 0,10 & 0,38 & 0,00 & 0,00 & 0,00 & 0,00 & 0,00 & 0,00 & 2 & 1 & & \\
\hline 512112 & 7,21 & 0,14 & 0,98 & 0,87 & 0,12 & 0,00 & 0,00 & 1,40 & 0,00 & 0,00 & 0,00 & 0,00 & 0,00 & 2 & 1 & & 1 \\
\hline 512113 & 0,24 & 0,71 & 1,96 & 1,84 & 0,28 & 0,00 & 0,00 & 0,00 & 0,00 & 0,00 & 0,00 & 0,00 & 0,00 & 2 & 1 & & \\
\hline 512190 & 4,60 & 0,40 & 1,11 & 0,46 & 0,08 & $0,, 13$ & 0,83 & 0,00 & 0,00 & 0,00 & 0,00 & 0,00 & 0,00 & 2 & 1 & & \\
\hline 512210 & 0,61 & 2,57 & 0,31 & 2,89 & 0,00 & 0,00 & 0,00 & 0,00 & 0,00 & 0,00 & 0,00 & 0,00 & 0,00 & 2 & 1 & & \\
\hline 519130 & 0,46 & 0,14 & 2,41 & 2,73 & 0,24 & 0,00 & 0,00 & 0,00 & 0,00 & 0,00 & 0,00 & 0,00 & 0,00 & 2 & 1 & & \\
\hline 541310 & 0,83 & 1,44 & 1,30 & 0,86 & 0,22 & 0,37 & 0,95 & 0,39 & 0,00 & 0,42 & 1,31 & 0,09 & 0,00 & 3 & 1 & & 1 \\
\hline 541320 & 0,48 & 1,07 & 1,10 & 2,82 & 0,00 & 0,00 & 4,09 & 0,00 & 0,00 & 0,00 & 0,00 & 1,64 & 0,00 & 5 & 1 & 1 & 1 \\
\hline 541340 & 0,87 & 0,53 & 1,73 & 0,56 & 0,62 & 0,10 & 1,01 & 4,17 & 0,00 & 0,00 & 6,37 & 0,00 & 0,00 & 4 & 1 & 1 & 1 \\
\hline 541410 & 0,49 & 0,81 & 0,79 & 0,30 & 0,24 & 0,08 & 0,79 & 0,55 & 15,62 & 2,36 & 2,42 & 0,00 & 0,00 & 3 & & & 1 \\
\hline 541420 & 0,45 & 0,45 & 1,38 & 0,32 & 0,20 & 1,92 & 10,29 & 0,00 & 0,00 & 0,00 & 0,00 & 3,43 & 0,00 & 4 & 1 & 1 & 1 \\
\hline 541430 & 0,51 & 0,83 & 0,73 & 0,88 & 1,78 & 1,26 & 2,49 & 0,38 & 0,23 & 2,82 & 1,11 & 0,47 & 0,00 & 5 & 1 & 1 & 1 \\
\hline 541490 & 0,26 & 1,00 & 2,25 & 0,11 & 5,49 & 0,80 & 0,49 & 1,68 & 0,00 & 0,00 & 0,00 & 0,00 & 0,00 & 4 & 1 & & 1 \\
\hline 541810 & 1,29 & 1,45 & 1,19 & 1,37 & 0,52 & 1,00 & 0,48 & 0,03 & 0,12 & 0,14 & 0,09 & 0,74 & 0,00 & 5 & 1 & 1 & \\
\hline 541820 & 2,58 & 1,44 & 0,42 & 2,44 & 0,00 & 0,00 & 0,15 & 0,00 & 0,00 & 0,00 & 0,00 & 0,00 & 0,00 & 4 & 1 & 1 & \\
\hline 541830 & 0,00 & 0,03 & 0,22 & 4,44 & 0,77 & 0,00 & 0,31 & 0,00 & 0,00 & 0,00 & 0,00 & 0,00 & 0,00 & 1 & 1 & & \\
\hline 541840 & 0,38 & 0,83 & 0,37 & 1,17 & 0,68 & 1,94 & 0,00 & 0,00 & 0,00 & 0,00 & 0,00 & 0,00 & 0,00 & 2 & 1 & 1 & \\
\hline 541850 & 1,81 & 0,56 & 0,66 & 1,41 & 0,57 & 2,72 & 1,75 & 0,00 & 0,90 & 2,11 & 0,00 & 0,00 & 0,00 & 5 & 1 & 1 & 1 \\
\hline
\end{tabular}

Continúa 


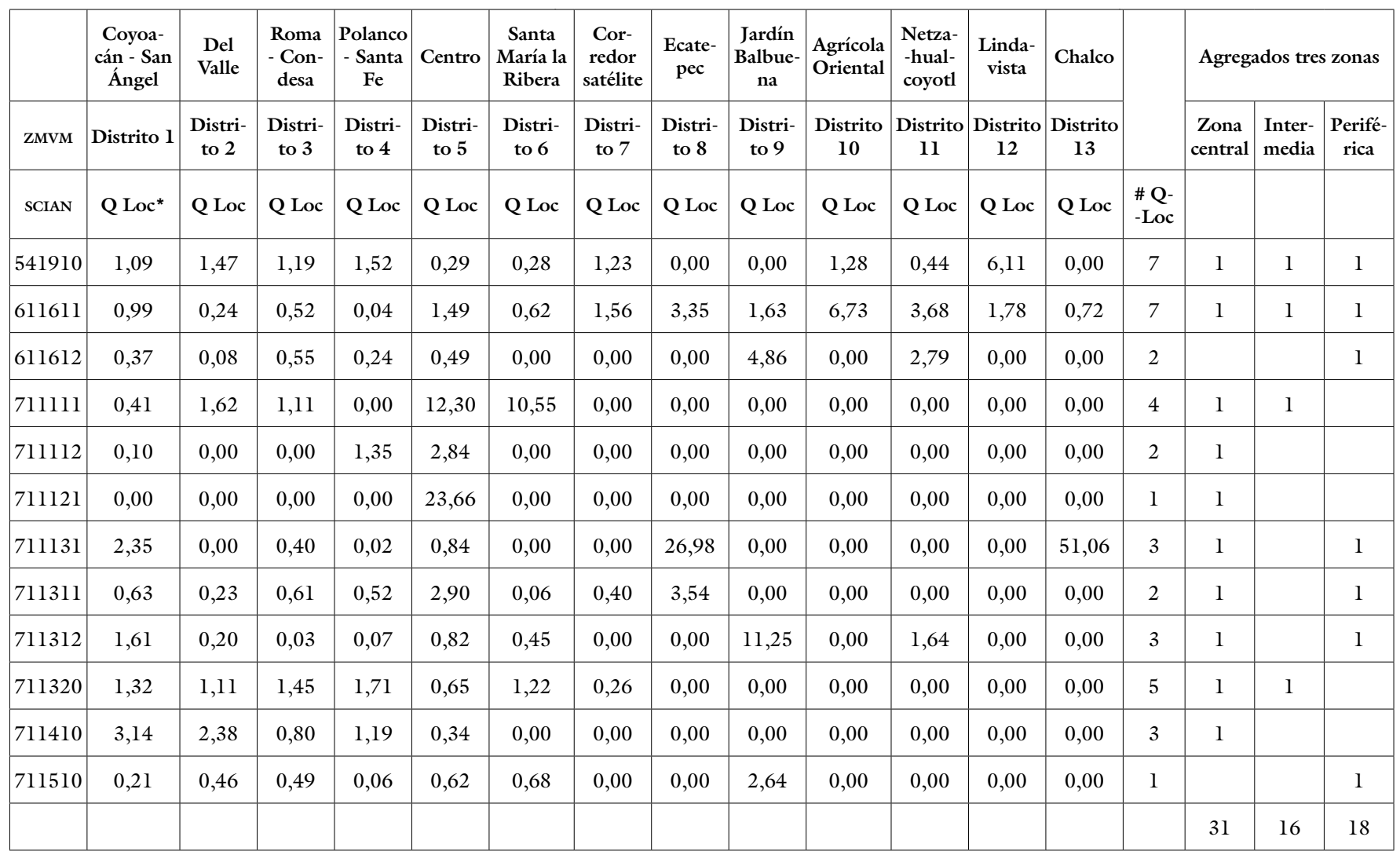

Fuente: elaboración propia con datos del DENUE 2012.

Finalmente, en el análisis de la estructura económica se compararon los 5 sectores principales de cada distrito. La información se obtuvo calculando el porcentaje de participación de las empresas en cada actividad económica a nivel de clase ( 6 dígitos) dentro de cada distrito. Se encontró que las estructuras de especialización son muy similares, ya que dominan los mismos sectores en la mayoría los distritos. 3 sectores se repiten en el 84,6\% de los distritos (Arquitectura 541310, Diseño Gráfico 541430 y escuelas de Arte privadas 611611), y el sector Agencias de Publicidad 541810 se repite en el $77 \%$ de los distritos.

Los datos presentados en esta sección indican que los distritos tienen más similitudes entre sí que diferencias y que es difícil hablar de distritos económicamente independientes. Hay otros elementos que apoyan esta tesis, diversos autores han analizado la distribución del empleo en la ZMVM, buscando encontrar evidencia de si la estructura urbana tiene un carácter territarios 34 207 
monocéntrico o policéntrico. Esta literatura es relevante para este análisis porque, por medio de sofisticadas metodologías han estudiado la concentración de empresas y empleo en el espacio urbano. Con estos estudios se puede contrastar la distribución que se encontró de empresas creativas y la distribución de las empresas en general. Los resultados de Suárez y Delgado revelan:

[...] una gran aglomeración central que se alarga al norte y al sur [...] a lo largo de Insurgentes y zonas adyacentes, otro de este a oeste a lo largo de Reforma llegando hasta Lomas-Santa Fe y una prolongación hacia Iztacalco-Iztapalapa teniendo como eje avenida Zaragoza (p. 2200).

El empleo está concentrado en las delegaciones centrales y a partir de ahí se extiende al sur y norte, este y oeste (Suarez \& Delgado, 2007). Varios estudios coinciden en esta gran "centralidad difusa" (Casado, 2012; Suárez \& Delgado, 2009), rasgo que se ratifica con el reciente estudio de Montejano (2015) que encuentra una estructura policéntrica muy débil en la Ciudad de México. La distribución mayoritaria de las empresas del sector creativo se ubica en esa misma centralidad difusa a la que aluden los especialistas en estructura urbana. Se puede concluir que no hay diferencia entre la ubicación de los servicios y el comercio en general y la ubicación intraurbana de las empresas creativas.

En cuanto a la segunda consideración territarias 34 208 sobre la relación entre el carácter urbano y el tipo de empresas, hay que decir que, como se refirió en la primer sección de este texto, una parte importante de la literatura sobre distritos creativos parten de casos $\mathrm{CO}^{-}$ nocidos o que en la opinión pública y académica se les percibe como casos aceptables de concentración de alguna actividad o con un carácter urbano (estético-cultural) que las distingue. En el caso de la Ciudad de México y su zona metropolitana, se puede decir que es aceptable hablar del Centro Histórico como un distrito creativo, al igual que lo es la zona de la Roma-Condesa, o la de Coyoacán-San Ángel. Pero hay que tener presente que la división que se ha realizado en 13 distritos creativos incluye zonas que difícilmente podrían ser designadas como creativas solo por su imagen y prestigio estético o cultural. Por otra parte, se demostró que los distritos ( 1 al 5), que componen la zona Central, si bien sí tienen características urbanas y son percibidos $\mathrm{co}^{-}$ mo zonas con carácter urbano particular en la imaginación de los habitantes de la Ciudad de México, comparten rasgos económicos significativos, por lo que en principio se puede cuestionar que haya un determinismo entre el tipo de empresas creativas y el contexto urbano donde se ubican.

\section{Conclusiones}

Los Clusters Económicos Creativos y los Distritos Urbanos Creativos hacen referencia a fenómenos espaciales y urbanos distintos y se manifiestan en escalas territoriales disímbolas. Es importante hacer explícita esta diferencia analíticamente, ya que la trasposición de los conceptos genera, 
desde nuestro punto de vista, apreciaciones erróneas sobre el funcionamiento de las industrias creativas. En términos de políticas públicas, para el desarrollo económico y de generación de empleo debe de tomarse en cuenta que las estrategias para el desarrollo de empresas creativas, y empresas/organizaciones de soporte a ellas, deben tener un alcance metropolitano y no limitarse a espacios restringidos dentro de la ciudad.

En términos empíricos, es importante resaltar los siguientes puntos. En primer lugar, los datos aportados indican que las industrias creativas tienen un papel relevante en la generación de empresas y empleo para la ciudad, por lo que se requiere de una mayor atención sobre su evolución y competitividad. En segundo lugar, Los datos analizados hasta aquí no sostienen la hipótesis de que los distritos sean suficientemente diferentes como para hablar de espacios económicos funcionales del tipo CEC. En todo caso, se puede hablar de una diferenciación entre las colonias de las zonas Centrales e Intermedias (distritos 1 al 7) y los distritos agrupados como zona periférica (8 al 13). En tercer lugar, los patrones de distribución intraurbana de los sectores creativos y el resto del comercio y servicios especializados, que reporta la literatura sobre subcentralidades urbanas en el ZMVM, son muy similares, por lo que no es posible asignar causalidad en la transformación urbana a este tipo de sectores, por el contrario, habría de buscar las condiciones institucionales y económicas que generan esta colocalización. Finalmente, las zonas de la Roma-Condesa, San Ángel-Coyoa- cán, o Polanco han capturado la imagen de ser espacios creativos; sin embargo, no se les puede entender como clusters económicos independientes, su dinámica está conectada con el resto de la ciudad. Tampoco son las únicas zonas de concentración de sectores creativos ni su condición como distritos urbanos depende solo de los sectores creativos. La identificación de aglomeraciones significativas de empresas creativas en zonas periféricas indica la posibilidad de impulsar el desarrollo de estos sectores en zonas de menores ingresos y no necesariamente cercanas a las zonas centrales de la ciudad o de altos ingresos.

\section{Referencias}

Alegría, T. (2009). Metrópolis Transfronteriza. Revisión de la hipótesis y evidencias de Tijuana, México y San Diego, Estados Unidos. México: COLEF-Porrua.

Arnaboldi, M., \& Spiller, N. (2011). Actornetwork theory and stakeholder collaboration: The case of Cultural Districts. Tourism Management, 32(3), 641-654.

Becattini, G. (1989). Sectors and/or districts: some remarks on the conceptual foundations of industrial economics. En E. Goodman, J. Bamford \& P. Saynor (Eds.), Small firms and Industrial districts in Italy. London y New York: Routledge.

Becattini, G. (2002). Del distrito Marshalliano a la contemporánea. Una breve reconstrucción crítica. Investigaciones regionales, $1(1)$, 9-32. territarios 34 209 
Brown, A., O'Connor, J., \& Cohen, S. (2000). Local music policies within a global music industry: cultural quarters in Manchester and Sheffield. Geoforum, 31(4), 437-451.

Casado Izquierdo, J. M. (2012). La estructura policéntrica de los mercados laborales locales de la Zona Metropolitana del Valle de México. Boletín del Instituto de Geografía, 2012(79), 97-118.

Chapple, K., Jackson, S., \& Martin, A. J. (2010). Concentrating creativity: The planning of formal and informal arts districts. City, Culture and Society, 1(4), 225-234.

Connolly, P. (2005). Tipos de poblamiento en la Ciudad de México. Observatorio Urbano de la Ciudad de México. Serie Metodología. México: UAM-Azcapotzalco.

Corredor Roma Condesa (2015). Web oficial del Corredor Cultural Roma Condesa. Recuperado de: http://www.ccromacondesa.mx.

Darchen, S. (2013). The Creative City and the Redevelopment of the Toronto Entertainment District: A BIA-Led Regeneration Process. International Planning Studies, 18(2) 188-203.

Delgadillo, V. (mayo, 2014). La política del espacio público y del patrimonio urbano en la ciudad de México. Discurso progresista, negocios inmobiliarios y buen comportamiento social. Ponencia presentada en XIII Coloquio Internacional de Geocrítica. Universidad de Barcelona, España.

territarias 34

De Propris, L., \& Wei, P. (2007). Governance and Competitiveness in the Bir- mingham Jewellery District. Urban Studies, 44(12), 2465-2486.

Florida, R., Mellander, C., \& Stolarick, K. (2010). Music scenes to music clusters: the economic geography of music in the US, 1970-2000. Environment and Planning A, 42(4), 785-804.

Florida, R., Mellander, C., \& Stolarick, K. (2011). Geographies of scope: an empirical analysis of entertainment, 19702000. Journal of Economic Geography, 12(1), 183-204.

Frost-Kumpf, H. A. (1998). Cultural Districts: The arts as a strategy for revitalizing our cities. Washington, D.C.: Americans for the arts.

Gómez H., S. (2015). Vivir en la Hipódromo la transformación del habitus como agente gentrificador. (Tesis de maestría, Universidad Autónoma de México, México, D.F.).

Homan, S. (2014). Liveability and creativity: The case for Melbourne music precincts. City. Culture and Society, 5(3), 149-155.

Hutton, T. A. (2010). The New Economy of the Inner City: Restructuring, Regeneration and Dislocation in the Twenty-first-century Metropolis. New York: Routledge.

Irani, D. (2014). Maryland Arts and Entertainment Districts Impact Analysis FY 2013. Maryland: Regional Economic Studies Institute.

Lazzeretti, L. (2008). El distrito cultural. En Soler, Vicente (Coord.), Los Distritos Industriales (pp. 330-351). España: Cajamar. 
Larrañaga, E. P. (2015). Mecanismos y dimensiones de transformación urbana en el modelo de la ciudad del conocimiento el caso Roma-Condesa. (Tesis de Maestría, UAM-Azcapotzalco, México, D.F.)

Lazzeretti, L. (Ed.). (2013). Creative industries and innovation in Europe. New York: Routledge.

López, L., \& Rebeca, B. (2012). La región: organización del territorio de la modernidad. Territorios, 27, 21-46.

Martin, R., \& Sunley, P. (2003). Deconstructing clusters: chaotic concept or policy panacea? Journal of Economic Geography, 3(1), 5-35.

McMillen, D. P. (2003). Employment subcenters in Chicago: Past, present, and future. Economic Perspective, 27(2), 2-14.

Mercado C. A. (2006). Pequeños exportadores $y$ desarrollo regional. La formación de capacidades locales en un distrito industrial mexicano. México: Plaza y Valdés.

Mercado C. A. (2011). El Cluster de la publicidad en la Ciudad de México. En A. Mercado \& M. Moreno (Eds.), La Ciudad de México y sus Clusters (pp. 95141). México: Juan Pablos-UAm Cuajimalpa.

Mercado C. A. (2012). Distritos culturales y empresas creativas en la Ciudad de México. Ponencia presentada en 1. ${ }^{a}$ Bienal Internacional Territorios en Movimiento, Universidad de Guanajuato, campus León.

Mizzau, L., \& Montanari, F. (2008). Cultural districts and the challenge of authenticity: the case of Piedmont, Italy. Journal of Economic Geography, 8(5), 651-673. doi:10.1093/jeg/lbn027

Mochi A., P. (2009). Los cluster tecnológicos en México y Argentina: una estrategia para el desarrollo local. Territorios 2021, 31-51.

Montejano, J. (2015). ¿Es la Ciudad de México policéntrica? Nuevos datos y herramientas SIG para la detección de centralidades urbanas. Revista Economía Sociedad y Territorio, XV(49), 333-361. Moreno, M., Mercado, A., \& Félix J. (2011). Producing, consuming and transforming the neighborhood: La Condesa in Mexico City. Ponencia presentada en International RC21 conference, Amsterdam Institute for Social Science Research - Urban Studies, University of Amsterdam. Ámsterdam, Holanda.

Municipio de Guadalajara (2014). Ciudad Creativa Digital. Recuperado de http://ccdguadalajara.com/es_ES/.

OECD. (2005). Culture and Local Development. Paris: OECD.

Pappalepore, I., Maitland, R., \& Smith, A. (2014). Prosuming creative urban areas. Evidence from East London. Annals of Tourism Research, 44(C), 227-240.

Porter, E. M. (1998). Clusters and the New Economics of Competition. Harvard Business Review, (Noviembre-Diciembre), 77-90.

Power, D., \& Scott, A. J. (2004). Cultural Industries and the Production of Culture. New York: Routledge.

Pratt, A. C. (2009). Urban Regeneration: From the Arts 'Feel Good' Factor to the Cultural Economy: A Case Study territarias 34 211 
of Hoxton, London. Urban Studies, 46(5-6), 1041-1061.

Richards, G. (2011). Creativity and tourism. Annals of Tourism Research, 38(4), 1225-1253.

Russo, A. P. (2000). The sustainable cultural cluster: notes on agglomeration, tourism policy and information technologies in tourist cities. Ponencia presentada en $40^{\text {th }}$ Congress of the European regional Science Association. European Regional Science Association. Barcelona, España. Sacco, P. L., Blessi, G. Tavano., Ferelli, G., \& Nuncio, M. (2008). Culture as an engine of local development processes: system-wide Cultural Districts. Art and Industrial Design. Growth and Change, 44(4), 555-570.

Santagata, W. (2002). Cultural districts, property rights and sustainable economic growth. International Journal of Urban and Regional Research, 26(1), 9-23.

Scott, A. (2005). On Hollywood. The Place, The Industry. Princeton, New Jersey: Princeton University Press.

Scott, A. J. (2001). Global City Regions. Trends, Theory, Policy. New York: Oxford University Press.

Scott, A. J. (2014). Beyond the Creative City: Cognitive-Cultural Capitalism and the New Urbanism. Regional Studies, 48(4), 1-14. doi:10.1080/00343404 .2014 .891010

Scott A. J., \& Lerriche F. (s. f.). Les ressorts ge 'ographiques de l'e 'conomie culturelle: du local au mondial. L'Espace Ge 'ographique, 34(3), 207-222.
Smit, A. J. (2011). The Influence of District Visual Quality on Location Decisions of Creative Entrepreneurs. Journal of the American Planning Association, 77(2), 167-184.

Stern, strangeM. J., \& Seifert, S. C. (2007). Cultivating "natural" cultural districts. Philadelphia: University of Pennsylvania.

Storper, M., \& Scott, A. (2003). Regions, Globalization, Development. Regional Studies, 37(6 \& 7), 579-593.

Storper, M., \& Venables, A. J. (2004). Buzz: face-to-face contact and the urban economy. Economic Geography, 4(4), 351 -370 .

Strange, W. C. (2008). Urban Agglomeration. En Steven N. Durlauf \& Lawrence E. Blume (Editores). The New Palgrave Dictionary of Economics. Basingstoke: Palgrave Macmillan.

Suarez L. M., \& Delgado C. J. (2007). Estructura y eficiencia urbanas. Accesibilidad a empleos, localización residencial e ingreso en la ZMCM 1990-2000. Economia, sociedad y territorio, 6(23), 693-724.

Suarez, M., \& Delgado, J. (2009). Is Mexico City Polycentric? A Trip Attraction Capacity Approach. Urban Studies, 46(10), 2187-2211.

Tironi, M. (2012). Enacting Music Scenes: Mobility, Locality and Cultural Production. Mobilities, 7(2), 185-210.

UNESCO y UNDP (2010). Creative Economy Report. New York: UNESCO.

Van den Berg, L., Braun, E., \& Van Winden, W. (2001). Growth clusters in Euro- 
pean cities: an integral approach. Urban Studies, 38(1), 185-205.

Van Heur, B. (2009). The Clustering of Creative Networks: Between Myth and Reality. Urban Studies, 46(8), 15311552 .

Zarlenga, M. I., Ulldemolins, J. R., \& Morato, A. R. (2013). Cultural clusters and social interaction dynamics: The case of Barcelona. European Urban and Regional Studies, 1, 1-19.
Zukin, S. (2009). Naked City: The Death and Life of Authentic Urban Places. Oxford: Oxford University Press.

Zukin, S., \& Braslow, L. (2011). The life cycle of New York's creative districts: Reflections on the unanticipated consequences of unplanned cultural zones. City, Culture and Society, 2(3), 131140. 
